https://doi.org/10.31426/ijamsr.2018.1.5.511

International Journal of

Advanced Multidisciplinary Scientific Research (IJAMSR) ISSN:2581-4281

\title{
A CONSERVATIVE APPROACH FOR ESTHETIC REHABILITATION OF AN ENDODONTICALLY TREATED MOLAR: A CASE REPORT
}

\author{
Dr Sakshi Trivedi ${ }^{1}$, Dr. Hunaid Arif Merchant ${ }^{2}$ \\ MDS- Conservative Dentistry and Endodontics, Ahmedabad, India. \\ MDS-Conservative Dentistry and Endodontics, Mumbai, India. \\ tsakshi21@gmail.com
}

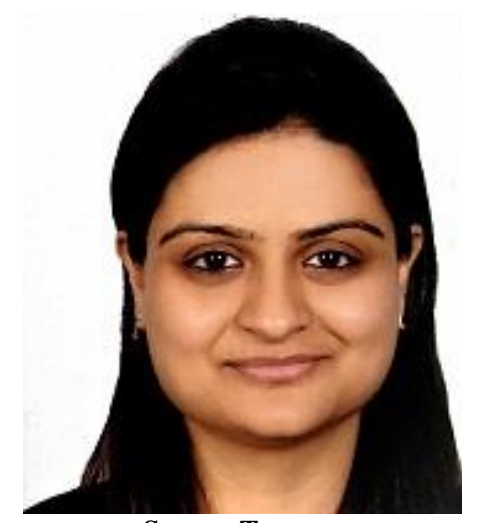

DR. SAKSHI TRIVEDI

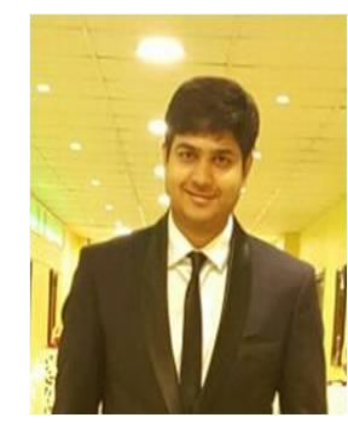

DR.HUNAID ARIF MERCHANT

\begin{abstract}
A B S T R A C T
Traditionally, most clinicians prefer to use posts of various types followed by crown restorations for the treatment of root filled posterior teeth. Nowadays, onlays and overlays have been proposed for restoring root filled teeth with promising results. Indirect ceramic overlays aim at the coverage of missing tooth structure with conservation of tooth structure, more importantly enamel which is required for adhesion and also a supragingival margin, which limits the irritation to the periodontium. These types of restorations permit greater control over contacts and reduce the negative effect of polymerization shrinkage when compared to direct composite restorations. Ribbond Polyethylene fibers have demonstrated their ability to withstand tensile stress and stop the crack propagation in composite material. This article presents a case report wherein an Endodontically treated molar was restored using packable composite alongwith resin fibre inserts and an occlusal coverage was provided using ceramic onlay.
\end{abstract}

Citation: Sakshi Trivedi, Hunaid Arif Merchant (2018). A Conservative Approach For Esthetic Rehabilitation Of An Endodontically Treated Molar: A Case Report. International Journal of Advanced Multidisciplinary Scientific Research (IJAMSR ) ISSN:2581-4281 Vol 1, Issue 5, July, 2018, \#Art.511, pp1-6

\section{Keywords:}

Conservative Approach,

Esthetic Rehabilitation,

Endodontically Treated Molar. 


\section{International Journal of Advanced Multidisciplinary Scientific Research (IJAMSR) ISSN:2581-4281}

Case

\section{Introduction}

During the last three decades, the development of the adhesive philosophy in dentistry has gradually changed the perception that a non vital tooth is equal to a crowned tooth and many classical indications for a crown restoration are nowadays questioned ${ }^{1}$. Traditionally, most clinicians prefer to use posts of various types followed by crown restorations for the treatment of root filled posterior teeth ${ }^{2}$. Composite restorations have also been evaluated for use in the restoration of root filled teeth. Recently, onlays and overlays have been proposed for restoring root filled teeth with promising in vitro results ${ }^{3}$. The fabrication of indirect ceramic or resin composite overlays or onlays aims at the coverage of missing tooth structure with limited further tooth preparation. These types of restorations permit greater control over occlusal and proximal contacts and reduce the negative effect of polymerization shrinkage when compared to direct restorations. In addition, 360-degree tooth preparation used in crown construction is avoided. A supragingival margin is easier to evaluate in situ, which consequently limits the irritation to the periodontium ${ }^{4}$.

The use of resin-based composite materials for the restoration of Endodontically treated posterior teeth has increased recently. Ribbond fibers are bondable, reinforced, ultrahigh strength polyethylene fibers. These fibers were reported to have a modifying effect on the interface stresses developed along the etched enamelresin boundary. By embedding a polyethylene fiber into the bed of a composite before restoration, higher microtensile bond strength could be achieved in prepared cavities with a high $\mathrm{C}$ factor ${ }^{5}$.
A 35 years old female patient reported to the Department of Conservative Dentistry and Endodontics with a chief complaint of incomplete Root canal treatment since last 15 days. At the examination root canal opening was done in relation to lower right first molar with a MOD cavity. Tenderness on percussion was negative, indicating the abscence of any periapical inflammation. Radiographic examination revealed no periapical changes and bone loss.

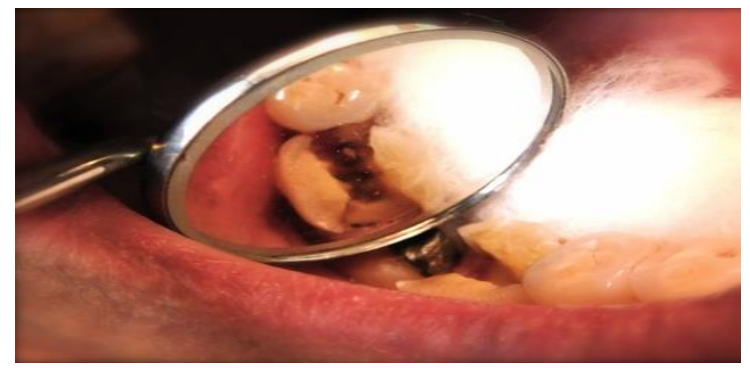

Fig:1- Preoperative photograph of lower right first molar showing incomplete root canal treatment

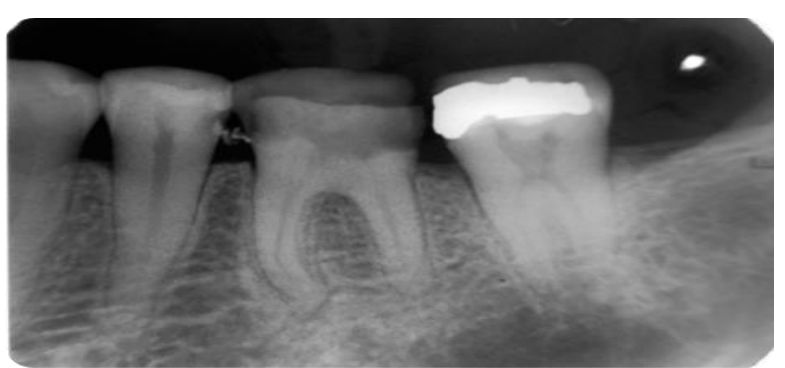

Fig:2- Preoperative radiograph of the tooth 
https://doi.org/10.31426/ijamsr.2018.1.5.511

I J A M S R

\section{International Journal of Advanced Multidisciplinary Scientific Research (IJAMSR) ISSN:2581-4281}

Procedure for Pulp space therapy:

Access cavity preparation was modified under rubber dam isolation followed by biomechanical preparation using rotary files (hyflex CM). Irrigation was done using saline and sodium hypochlorite $(3 \%)$ alternatively. The canal was dried using paper points and obturation was done using the lateral condensation technique.

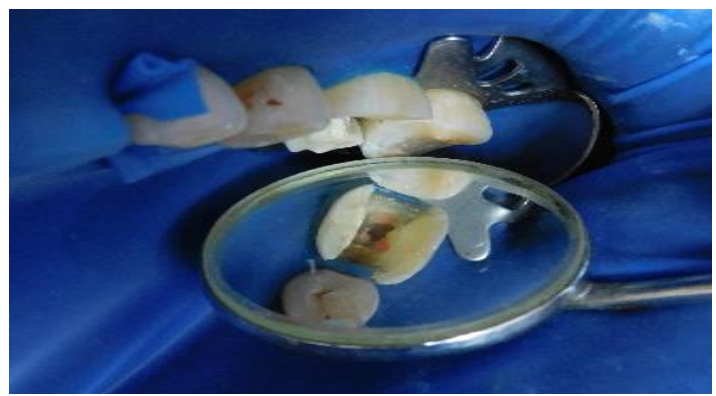

Fig3- post obturation photograph

Procedure for core build up:

Acid etching was carried out using 37\% phosphoric acid for 15 seconds. Acid was rinsed and the tooth was dried using chip blower. Two coats of bonding agent was applied in thin layers and light cured for 20 seconds. Ribbondfibres were cut according to the pre-measured size of the cavity walls and pulpal floor and placed in the following fashion: One piece was placed on the pulpal floor extending on the buccal and lingual walls axially. Two pieces were embedded on the mesial and distal walls build with composite. These fibre pieces were fixed into position and cured along with a thin layer of flowable composite, which acted like a stress breaker.

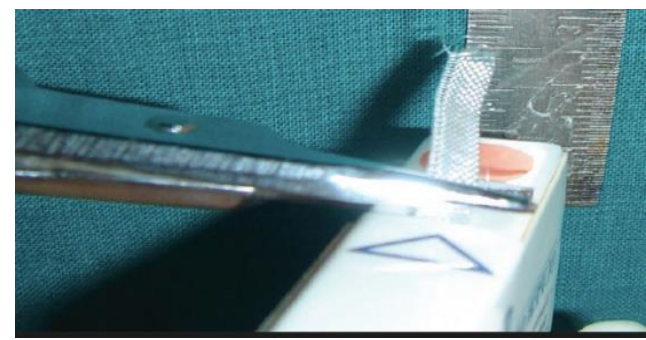

Fig4- Ribbondfibre cut into premeasured size

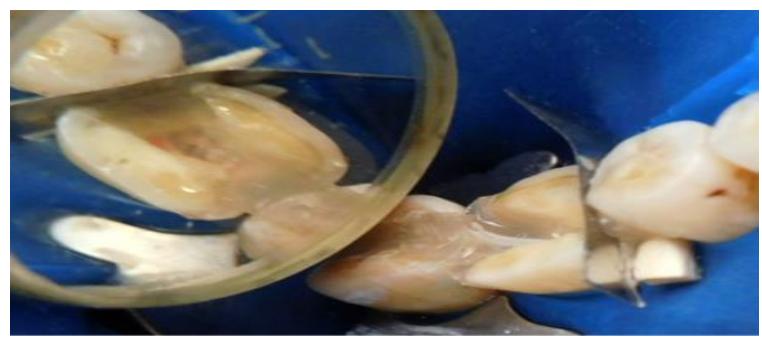

Fig5- Fibre being placed alongwith a layer of flowable composite on the pulpal floor.

Incremental core build- up was done using packable composite material. Finishing and polishing procedures were achieved using aluminium oxide discs. Thecuspal coverage was obtained with a minimal invasive reduction of the buccal and lingual cusps and restoration margins are placed in the incisal third at a distance of 2 $\mathrm{mm}$ from the occlusal plane and $1.5 \mathrm{~mm}$ from the lateral contacts. A thick band of enamel on the margins was preserved, which would aid in superior adhesion. Impression was made using addition silicone material and cast was poured and sent to laboratory for lithium disilicate (IPS empress) onlay fabrication. 


\section{https://doi.org/10.31426/ijamsr.2018.1.5.511 \\ International Journal of I J A M S R Advanced Multidisciplinary Scientific Research (IJAMSR) ISSN:2581-4281}

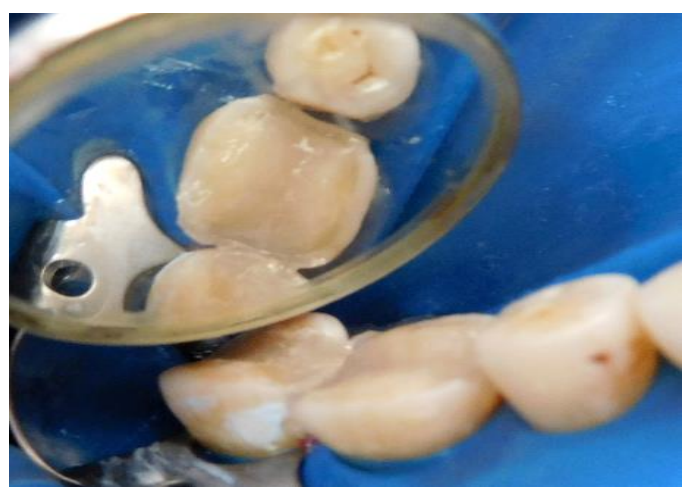

Fig 6- Core build up done using packable composite

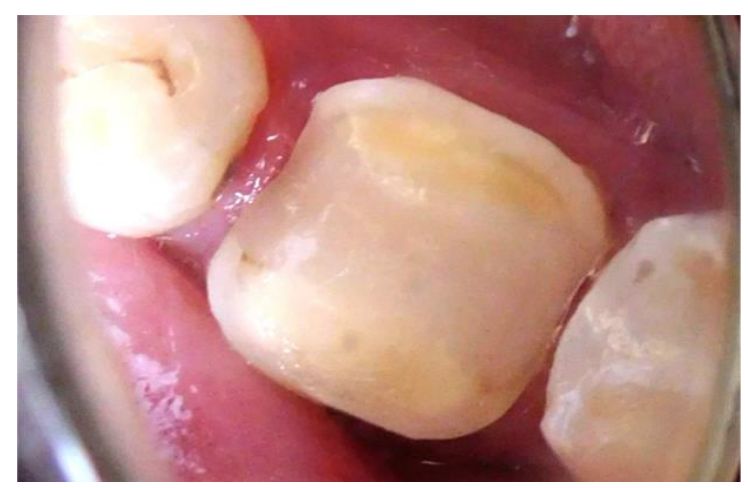

Fig 7- Tooth surface prepared for ceramic overlay

\section{Procedure for Cementation}

Adhesive preparation of the tooth- $37 \%$ orthophosphoric acid was used to etch enamel for 15 seconds followed by rinsing with distilled water and drying using chip blower. Dentin bonding agent (supplied along with multilink resin cement) was applied onto the etched enamel and composite resin and cured for 20 seconds.

Adhesive preparation for the ceramic onlay- The intaglio surface of ceramic onlay was treated with hydrofluoric acid following manufacturer's instructions. The restoration was abundantly rinsed with water spray and dried using air jet. A silane-coupling agent (Monobond plus; ivoclarvivadent) is applied on the dried surface. Dual cured resin cement (multilink; ivoclarvivadent) is dispensed onto the inner surface of the restoration and placed onto the prepared tooth surface with slight pressure. Full polymerization is achieved by light curing for at least 90 seconds per surface.

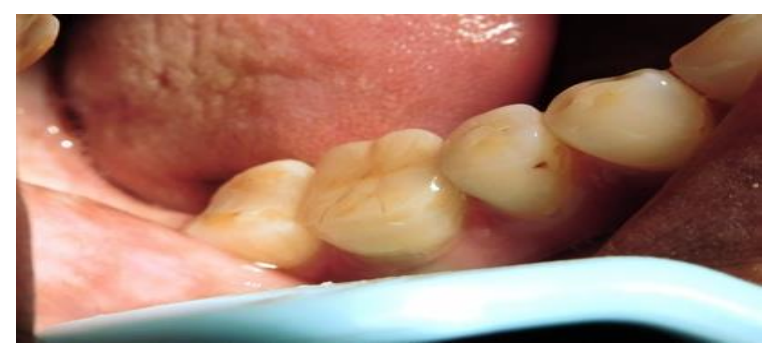

Fig 8- Postoperative photograph after overlay cementation

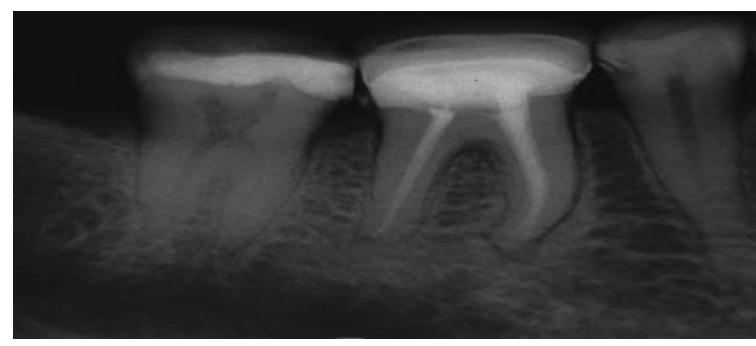

Fig 9- Postoperative radiograph of the tooth

Discussion

The anatomical and physiological differences between molars, premolars \&anteriors play a major role while choosing the type of restoration for root canal treated teeth. Firstly, molars have larger pulp chambers as compared to premolars and anteriors thus providing greater surface area for adhesive procedures. Secondly, in a normal occlusal context; posterior teeth are more subjected to axial forces while anterior teeth are more exposed to shear forces. Thus, in most cases molars can 


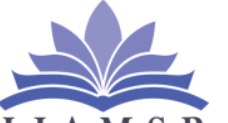

I J A M S R

\section{International Journal of Advanced Multidisciplinary Scientific Research (IJAMSR) ISSN:2581-4281}

be restored by relying solely on adhesion, while premolars \&anteriors might require a post placement in

\section{Conclusion}

case of excessive loss of tooth structure. In the coronal part of the tooth, a few particular cavity configurations seem to be more at risk. Studies show that the marginal ridge preservation is elemental in limiting abnormal cuspal deflection and fracture. Thus, an occlusal cavity and a mesial-occlusal-distal cavity are on the opposite side of a hypothetical safety scale. As a consequence, the MOD cavities on should be considered as having the worst prognosis in terms of fracture risk. In such cases, a cuspal coverage of the tooth is recommended in order to avoid extreme flexure of the cusps ${ }^{6}$.

Bonded restorations with full occlusal coverage or "overlays" have a beneficial effect on fracture strength of root canal treated teeth as compared to simple MOD restorations 7,8 . Bonded overlays show a more homogeneous distribution of masticatory forces and have a better cavity configuration in terms of $\mathrm{C}$ factor, which allows lesser polymerization shrinkage stresses on adhesive interfaces ${ }^{8}$. When fiber inserts are placed in composite restorations, they increase the quality of the marginal zone of restoration by replacing the part of the composite increment, which in turn reduces the volumetric polymerization shrinkage of the composite. The fibers also may have a strengthening effect on the composite margin, which may increase resistance to dimensional change or deformation that occurs during thermal and mechanical loading thus, improving marginal adaptation 9. A layer of flowable composite was used on the pulpal floor because low elasticity modulus of flowable composites provides flexibility for the bonded restoration. Liners act as stress breakers and aid in equal distribution of stresses over the adhesive interface ${ }^{10}$.
This case report illustrates how the principles of minimally invasive dentistry can be applied in the restoration of Endodontically treated teeth. Weakened posterior teeth can be restored functionally and esthetically by direct and indirect adhesive techniques, thus avoiding a further sacrifice of sound tissues and almost eliminating the use of posts on non vital molars. Finally, it is important to note that accurate case selection, treatment planning and following strict protocols for adhesive procedures are essential for the accomplishment of this conservative strategy.

\section{References}

1) Krejci I, Duc O, Dietschi D, de Campos E. Marginal adaptation, retention and fracture resistance of adhesive composite restorations on devital teeth with and without posts. Oper Dent 2003;28:127-135.

2) Naumann M, Kiessling S, Seemann R. Treatment concepts for restoration of endodontically treated teeth: a nationwide survey of dentists in Germany. $J$ Prosthet Dent 2006;96:332-8

3) Jiang W, Bo H, Yongchun G, Longxing N. Stress distribution in molars restored with inlays or onlays with or without endodontic treatment: a threedimensional finite element analysis. J Prosthet Dent 2010;103:6-12.

4) Blank JT. Scientifically based rationale and protocol for use of modern indirect resin inlays and onlays. $J$ EsthetRestor Dent 2000;12:195-208.

5) Belli $S$, Donmez, N, \&Eskitascioglu $G$. The effect of $C$ factor and flowable resin or fiber use at interface on microtensile bond strength to dentin. J Adhes Dent 2006;8(4):247-253. 
https://doi.org/10.31426/ijamsr.2018.1.5.511

I J A M S R

International Journal of Advanced Multidisciplinary Scientific Research (IJAMSR) ISSN:2581-4281

6) Rocca GT, Krejci I. Crown and post-free adhesive restorations for endodontically treated posterior teeth: from direct composite to endocrowns. Euro J Esthet Dent 2013;8(2):156-179.

7) Adolphi G, Zehnder M, Bachmann LM, Göhring TN. Direct resin composite restorations in vital versus root-filled posterior teeth: a controlled comparative long-term follow-up. Oper Dent 2007;32:437-442.

8) Bitter K, Meyer-Lueckel H, Fotiadis N, Blunck U, Neumann K, Kielbassa AM, Paris S. Influence of endodontic treatment, post insertion, and ceramic restoration on the fracture resistance of maxillary premolars.IntEndod J 2010;43:469-477.

9) Xu HH, Schumacher GE, Eichmiller FC, Peterson RC, Antonucci JM \& Mueller HJ. Continuous-fiber preform reinforcement of dental resin composite restorations. Dent Mater J 2003;19(6): 523-530.

10) Beznos C. Microleakage at the cervical margin of composite Class II cavities with different restorative techniques. Oper Dent 2001; 26(1):60-69. 